\title{
Diagnosis of adult ALCAPA with computed tomography coronary artery
}

\author{
Yew Eng Tan ${ }^{1}$, Kok King Chia ${ }^{1}$ and Noor Khairiah Abdul Karim ${ }^{2 *}$ (])
}

\begin{abstract}
Background: Anomalous left coronary artery from the pulmonary artery (ALCAPA) is a rare congenital anomaly where the left main coronary artery arises from the pulmonary artery, instead of the coronary sinus of the ascending aorta. ALCAPA is divided into infant and adult types. Life-threatening complications such as malignant arrhythmia and sudden death could ensue in adult type of ALCAPA. Imaging is the current preferred modality for ALCAPA. It is indeed a challenge to diagnose ALCAPA due to its non-specific clinical presentation and laboratory findings. We report a case of adult type ALCAPA presented with unstable angina surprisingly surviving into adulthood with no symptoms prior to presentation, which is extremely rare.
\end{abstract}

Case presentation: A 53-year-old lady presented with sudden onset of chest pain and worsening shortness of breath. She was initially treated as unstable angina. Physical examination revealed normal heart sound. Computed tomography coronary angiography (CTCA) showed an anomalous origin of the left coronary artery from the posterior wall of the proximal pulmonary artery, compatible with ALCAPA. The anomalous left coronary artery bifurcates into left anterior descending and left circumflex arteries. Cardiothoracic surgeon planned for occlusion of ALCAPA via the pulmonary artery, owing to the fact that unfeasible rerouting in the presence of well-established collateral supply.

Conclusions: ALCAPA is a rare and life-threatening condition in adults which may lead to myocardial infarction and sudden death in untreated cases. CTCA is one of the preferred modern imaging modality in ALCAPA owing to its superior ability for direct visualization of the anomaly. Hence, early identification and surgical intervention of the anomaly are paramount to reduce the morbidity and mortality.

Keywords: Congenital anomaly, Adult onset ALCAPA, Computed tomography coronary angiography

\section{Background}

Anomalous left coronary artery from the pulmonary artery (ALCAPA) is a rare congenital anomaly where the left main coronary artery arises from the pulmonary artery (PA), instead of the coronary sinus of the ascending aorta. It has an incidence of 1 in 300,000 births [1]. It is indeed a challenge to diagnose ALCAPA due to its non-specific clinical presentation and laboratory findings. Herein, we report a case of adult type ALCAPA

\footnotetext{
*Correspondence: drkhairiah@usm.my

${ }^{2}$ Regenerative Medicine Cluster, Advanced Medical and Dental Institute, Universiti Sains Malaysia, Bertam, 13200 Kepala Batas, Pulau Pinang, Malaysia

Full list of author information is available at the end of the article
}

presented with unstable angina surprisingly surviving into adulthood with no symptoms prior to presentation, which is extremely rare.

\section{Case presentation}

A 53-year-old lady presented with sudden onset of chest pain and shortness of breath. Prior to this presentation, she had reduced effort tolerance for a duration of 3 months. She had difficulty in completing her usual daily activities due to reduced effort tolerance, and difficulty falling into sleep at night due to mild shortness of breath. However, she perceived that as normal and did not seek for medical attention until she developed worsening of shortness of breath. She had no significant 
past medical or surgical history, and unremarkable childhood and adulthood. She was able to carry out regular physical activities. She had four uneventful spontaneous vaginal deliveries. Physical examination revealed normal heart sounds without heart murmur. There was no pedal oedema. Electrocardiography (ECG) showed sinus rhythm and mild ST depression in lateral leads (V5 and V6). She was initially treated as unstable angina.

Echocardiography was performed immediately on the same day after admission and showed abnormal turbulent flow between right coronary artery (RCA) and right ventricle, suspicious of a RCA fistula (Fig. 1). Subsequent CTCA showed an anomalous origin of the left coronary artery from the posterior wall of the proximal PA, compatible with ALCAPA (Fig. 2). The anomalous left coronary artery bifurcates into left anterior descending and left circumflex arteries. Multiple well-established collateral arteries were noted. No atherosclerosis or arterial stenosis were identified. Conventional coronary angiography and right heart study that were performed a week later demonstrated the ALCAPA with single coronary arterial supply via the dilated RCA with left-to-right shunt.

She was managed conservatively with medication and upon discharged, she was given antihypertensives, diuretic, anticoagulant and phosphodiesterase inhibitors. She remained well and asymptomatic for the past one year with regular follow-up in the outpatient clinic. She

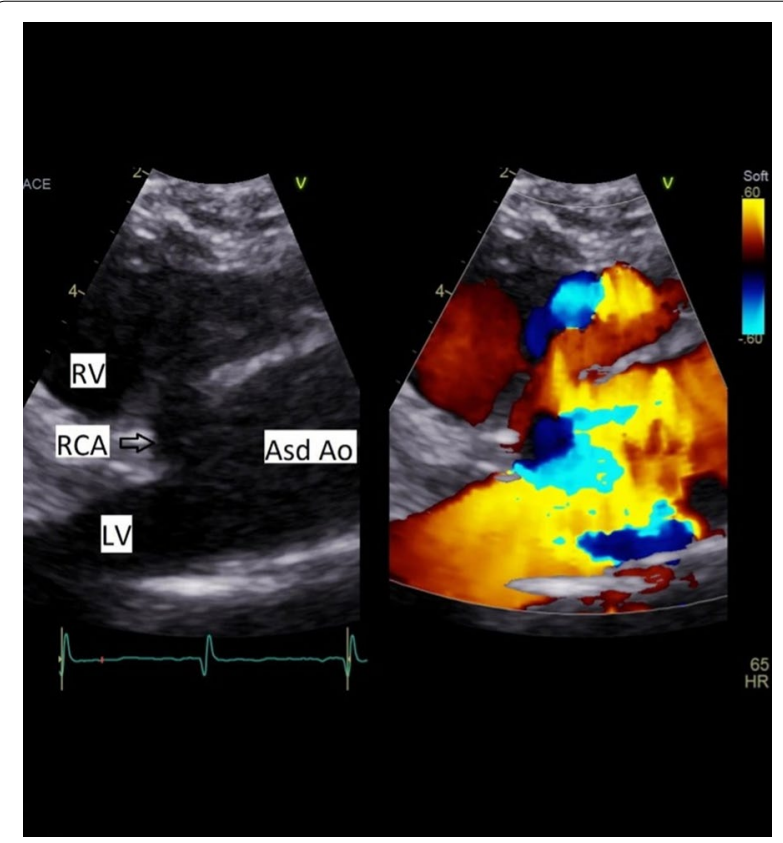

Fig. 1 Echocardiography shows turbulent flow by colour flow mapping over right coronary artery (RCA) into the right ventricle (RV), suspicious of a RCA fistula

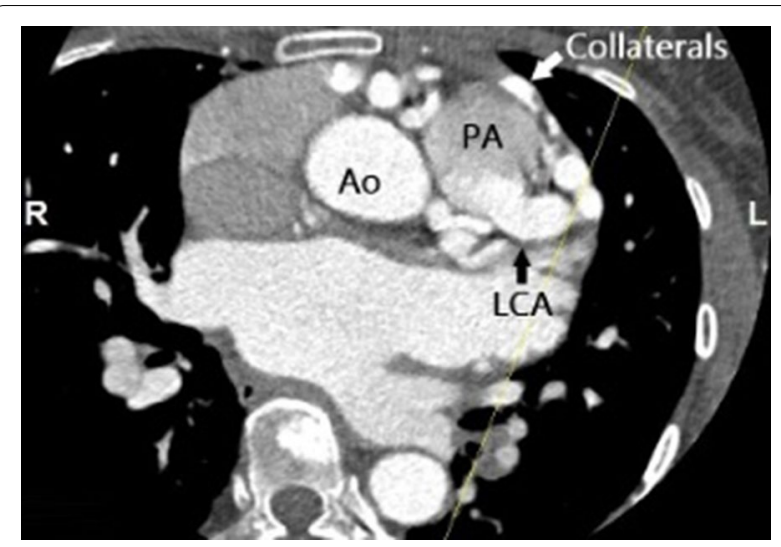

Fig. 2 CTCA confirming the origin of the left coronary artery (LCA) arising from the pulmonary artery (PA) and not from the aorta (Ao). Intercoronary collateral arteries are seen along the external surface of the heart

is currently under cardiothoracic surgical department follow-up, awaiting surgery to be done. Cardiothoracic surgeon planned for occlusion of ALCAPA via the PA, owing to the fact that unfeasible rerouting in the presence of well-established collateral supply. No further cardiovascular imaging was planned.

\section{Discussion}

ALCAPA or Bland-White-Garland syndrome is a rare and life-threatening congenital anomaly where the left coronary artery arises from the PA instead of the aortic sinus. This leads to a left-to-right shunt, resulting in "coronary steal" and impaired coronary arterial supply. It was first reported in 1885 by Brooks in Dublin. The first clinical description in conjunction with autopsy findings was described by Bland and colleagues in 1933.

This anomaly accounts for 1 in 300,000 births [1]. There are two types of ALCAPA: the infant type and adult type, each of which has different manifestations and outcomes. Approximately $90 \%$ die within the first year of life in infant type due to myocardial infarction and congestive heart failure [2]. In adult type, they may be asymptomatic for years, however if this anomaly is not corrected, patients may develop progressive cardiac dysfunction, malignant arrhythmia, myocardial infarction and eventually sudden death. This has an estimated incidence rate up to $90 \%$ at the mean age of 35 years [3].

ECG findings are not specific and may show evidence of ischemia with presence of Q waves up to $50 \%$ of patients, $28 \%$ with left ventricular hypertrophy and 4\% with normal ECG respectively [4]. Echocardiography may detect a dilated RCA, retrograde Doppler flow from left coronary artery to PA and visualization of septal flow due to collaterals [4]. However, echocardiography 
is operator-dependent and may not be easily recognized due to lack of knowledge on this entity and the pitfall of echocardiography because it is a two-dimensional imaging.

Conventional coronary angiography used to be the gold standard to detect coronary anomalies prior to the development of the CTCA and magnetic resonance imaging (MRI). Today, different imaging techniques have changed the usual daily practice to diagnose coronary anomalies. The technology advancement has substantially increased the detection of this anomaly in adults [4]. CTCA and MRI are currently the preferred modalities for those with suspected ALCAPA, as well as on preoperative planning and postoperative follow-up. Hence, the identification of this condition has increased and leading to major changes in the management and survival [5].

CTCA offers excellent spatial resolution and volume rendering technique to visualize the coronary arteries. The radiographic features in CTCA for ALCAPA include direct visualization of the origin of the left coronary artery from the posterior aspect of the PA, dilated and tortuous RCA, and dilated intercoronary collateral arteries along the external surface of the heart or within the interventricular septum [4]. MRI has the advantage of physiological and functional assessment in cardiac anomalies. In ALCAPA, it can be used to assess the systolic function of the left ventricle, visualization of the communication and the retrograde flow from the left coronary artery to the PA and myocardial viability [4].

The treatment of choice for this anomaly is surgical intervention to restore a dual-coronary-artery system [1] which include left coronary artery ligation with or without coronary artery bypass grafting, the Takeuchi procedure namely direct anastomosis of the anomalous left coronary artery from the PA directly to the aorta. Another optional procedure that had been described in recent literature with high success rate was occlusion of the ALCAPA using detachable patent ductus arteriosus coil and ventricular septal defect occluder [6, 7]. In our case, while awaiting for occlusion of ALCAPA via the PA to be performed, she is currently managed conservatively and has remained well.

\section{Conclusions}

ALCAPA is a rare and life-threatening condition in adults which may lead to myocardial infarction and sudden death in untreated cases. CTCA is one of the preferred modern imaging modality in ALCAPA owing to its superior ability for direct visualization of the anomaly. Hence, early identification and surgical intervention of the anomaly are paramount to reduce the morbidity and mortality.

\section{Abbreviations}

ALCAPA: Anomalous left coronary artery from the pulmonary artery; RCA: Right coronary artery; CTCA: Computed tomography coronary angiography; ECG: Electrocardiography; PA: Pulmonary artery; MRI: Magnetic resonance imaging.

\section{Acknowledgements}

The authors thank the study participant for the support and help.

\section{Authors' contributions}

Radiological findings were reported by TYE and CKK. The case report writeup was prepared by TYE and supervised by NKAK. All authors read and approved the final manuscript.

\section{Funding}

Not applicable.

\section{Availability of data and materials}

The data are taken solely from our institution.

\section{Declarations}

Ethics approval and consent to participate

A written informed consent was obtained from the patient.

\section{Consent for publication}

A written informed consent for participation was obtained from the patient.

\section{Competing interests}

We have no competing interests to declare.

\section{Author details}

${ }^{1}$ Diagnostic Imaging Department, Hospital Sultanah Bahiyah, Km 6, Jalan Langgar, Bandar Alor Setar, 05460 Alor Setar, Kedah, Malaysia. ${ }^{2}$ Regenerative Medicine Cluster, Advanced Medical and Dental Institute, Universiti Sains Malaysia, Bertam, 13200 Kepala Batas, Pulau Pinang, Malaysia.

Received: 20 September 2021 Accepted: 21 November 2021

Published online: 30 November 2021

\section{References}

1. Pachon R, Bravo C, Niemiera M (2014) Anomalous origin of the left coronary artery from pulmonary artery (ALCAPA). J Clin Exp Cardiology. https://doi.org/10.4172/2155-9880.1000341

2. Peña E, Nguyen ET, Merchant N, Dennie C (2009) ALCAPA syndrome: not just a pediatric disease. Radiographics. https://doi.org/10.1148/rg. 292085059

3. Zacharias M, Chandok D, Tighe D (2015) A late presentation of an anomalous left coronary artery originating from the pulmonary artery (ALCAPA): a case study and review of the literature. J Cardiol Cases. https://doi.org/10.1016/j.jccase.2014.10.006

4. Yau JM, Singh R, Halpern EJ, Fischman D (2011) Anomalous origin of the left coronary artery from the pulmonary artery in adults: a comprehensive review of 151 adult cases and a new diagnosis in a 53-year-old Woman. Clin Cardiol. https://doi.org/10.1002/clc.20848

5. Doris MK, Newby DE (2016) How should CT coronary angiography be integrated into the management of patients with chest pain and how does this affect outcomes? Eur Heart J Eur Heart J Quality Care Clin Outcomes 2(2):72-80. https://doi.org/10.1093/ehjqcco/qcv027

6. Sagar P, Sivakumar K (2021) Transcatheter occlusion of an anomalous origin of left coronary artery from pulmonary artery in an adult as an 
alternative to surgery. Cardiol Young. https://doi.org/10.1017/S1047 951120003418

7. Bimal F, Harikrishnan S, Titus T, Tharakan JM (2002) Percutaneous coil closure of recanalised anomalous origin of left coronary artery from pulmonary artery. Int J Cardiol. https://doi.org/10.1016/S0167-5273(02) 00054-2

\section{Publisher's Note}

Springer Nature remains neutral with regard to jurisdictional claims in published maps and institutional affiliations.

\section{Submit your manuscript to a SpringerOpen ${ }^{\circ}$ journal and benefit from:}

- Convenient online submission

- Rigorous peer review

- Open access: articles freely available online

- High visibility within the field

- Retaining the copyright to your article

Submit your next manuscript at $\boldsymbol{\nabla}$ springeropen.com 